\title{
The role of klotho in systemic sclerosis
}

\author{
R. Talotta ${ }^{1}$, S. Bongiovanni', T. Letizia ${ }^{2}$, F. Rigamonti' ${ }^{1}$, F. Atzeni', \\ M. Benucci ${ }^{3}$, T. Vago ${ }^{2}$, P. Sarzi-Puttini ${ }^{1}$ \\ ${ }^{1}$ Department of Rheumatology, Luigi Sacco University-Hospital, Milan; \\ Endocrinology and Rheumatology Laboratory, Luigi Sacco University-Hospital, Milan; \\ ${ }^{3}$ Department of Rheumatology, San Giovanni di Dio Hospital, Florence, Italy
}

\section{SUMMARY}

The aim was to evaluate the role of klotho in the pathogenesis of systemic sclerosis (SSc), through the measurement of its serum concentration in SSc patients compared to healthy controls, and to assess the association with cutaneous and visceral involvement.

Blood samples obtained from both SSc patients and healthy controls were analysed by an ELISA assay for the detection of human klotho. SSc patients were globally evaluated for disease activity and assessed through the modified Rodnan's Skin Score, Medsger's scale, pulmonary function tests, 2D-echocardiography, nailfold capillaroscopy and laboratory tests.

Our cohort consisted of $69 \mathrm{SSc}$ patients (61 females, mean age $64.5 \pm 12.5$ years, median disease duration 9.0 (IQR 8) years) and 77 healthy controls (28 females, mean age $49.7 \pm 10.2$ years). In the group of SSc patients, $19(27.5 \%)$ suffered from a diffuse form of SSc. All patients were receiving IV prostanoids, and some of them were concomitantly treated with immunosuppressive drugs (prednisone, hydroxychloroquine, mofetil mycophenolate, methotrexate, cyclosporin A and azathioprine). The median serum concentration of klotho was significantly lower in patients compared to controls $(0.23 \mathrm{ng} / \mathrm{mL}$ vs $0.60 \mathrm{ng} / \mathrm{mL} ; \mathrm{p}<0.001)$. However, Spearman's test showed no significant association between klotho serum levels and disease activity, concerning either clinical, laboratory or instrumental findings.

Our data show a significant deficit of klotho in SSc patients although any significant association was detected between klotho serum concentration and the clinical, laboratory or instrumental features of the disease. However, due to the limits of the study, further investigations are required.

Key words: Systemic sclerosis; klotho; biomarkers.

Reumatismo, 2017; 69 (4): 156-163

\section{INTRODUCTION}

ystemic sclerosis $(\mathrm{SSc})$ is a connec$\checkmark$ tive tissue disease characterized by a microvessel dysfunction, the aberrant activation of the immune system, and the deposition of extracellular matrix in skin and viscera. Endothelial cells and contractile myofibroblasts, involved in microvessel dysfunction, contribute to the first step in the pathogenesis of the disease, whereas the immune system may play a secondary role. Klotho is a trans-membrane, soluble and secreted protein that displays enzymatic and co-receptor functions, being involved in the prevention of aging, in the calcium-phosphate balance and in the reparative and fibrotic processes $(1,2)$. Transgenic klotho-/- mice develop a phenotype characterized by accelerated aging, atherosclerosis, hyper-phosphatemia, osteoporosis, cutaneous atrophy and emphysema and have a lower likelihood in cutaneous wound healing (3). As klotho seems to play an essential role in the maintenance of vessel tone control, in tissue regeneration and reparation and in the electrolytic balance, a deficit in the concentration of this molecule could explain some phenotypic aspects of SSc, like microvessel dysfunction, calcinosis and fibrosis.

The primary objective of this study was to evaluate klotho as a biomarker of SSc, to measure its concentration as a soluble molecule in the peripheral blood of SSc patients and to compare it with healthy controls. The secondary objective was to find any significant association with clinical, laboratory and instrumental manifestations of the disease. 


\section{MATERIALS AND METHODS}

Sixty-nine consecutive SSc patients, according to the 2013 EULAR/ACR criteria (4), and 77 healthy controls were enrolled in our study. After signing an informed consent, venous blood samples obtained from both patients and controls were collected and centrifuged. Sera were analysed through an ELISA assay, aiming to detect the human soluble klotho by means of a monoclonal anti-KL antibody and a KLHRP conjugate (My Biosource, CA, USA), with a sensitivity of $0.1 \mathrm{ng} / \mathrm{mL}$.

SSc patients were assessed for the most specific clinical manifestations (including Raynaud's phenomenon, digital ulcers, calcinosis), through the Medsger's scale score (5) for the severity of global visceral involvement and through the modified Rodnan's Skin Score (mRSS) for cutaneous involvement. Moreover, the demographic and clinical data concerning pulmonary function tests (forced vital capacity or FVC; diffusing capacity/transfer factor of the lung for carbon monoxide per unit alveolar volume or DLCO/AV; chest high resolution computer tomography or HRCT scan), cardiac indexes (2D echocardiography or 2D-ECHO) as indirect estimator of the risk of pulmonary artery hypertension $(\mathrm{PAH})$, nailfold capillaroscopic pattern and laboratory tests (serum creatinine level; erythrocyte sedimentation rate or ESR; $\mathrm{C}$-reactive protein or CRP; autoantibodies), as well as concomitant pharmacologic treatments were also recorded for every single patient.

Since klotho may be involved in the prevention of diabetes, renal failure and bronchial obstructive disease, these disorders were considered as exclusion criteria.

\section{Statistical analysis}

Parametric and non-parametric statistical tests according to data distribution have been used. When data were parametrically distributed, results were reported as mean \pm standard deviation (SD); when data were not parametrically distributed, we reported the results as median, interquartile interval (IQ) and range. A two-tailed Mann Whitney $U$ test for unpaired samples was used to compare the serum concentration of klotho between patients and controls. In the group of SSc patients, Spearman's test was used to correlate the level of klotho with clinical, laboratory and instrumental findings. Statistical analysis was carried out using a SPSS calculator, version 23, setting the $\mathrm{P}$-value at $<0.05$.

\section{RESULTS}

Sixty-nine consecutive SSc patients (61 females, mean age \pm SD $64.5 \pm 12.5$ years, median (IQ) disease duration 9.0 (8) years) and 77 healthy controls ( 28 females, mean age \pm SD $49.7 \pm 10.2$ years) were enrolled in the study. In the group of SSc patients, $19(27.5 \%)$ suffered from a diffuse form of disease. Antinuclear antibodies (ANA) were detected in $64(92.7 \%)$ patients, anticentromere (anti-CENB) autoantibodies in $34(49.2 \%)$ patients and anti-Scl70 autoantibodies in 17 (24.6\%) patients. In order to counteract Raynaud's phenomenon and prevent digital ulcers, all patients were receiving intravenous (IV) prostanoids, and 21 patients were concomitantly treated with calcium channel blockers (CCBs). Seven patients were taking bosentan for the treatment of PAH and the prevention of digital ulcers. None of the patients were treated with phosphodiesterase-5 inhibitors. In addition, several patients were also treated with immunosuppressive drugs (prednisone $2.5-5 \mathrm{mg} /$ day in 5 cases, hydroxychloroquine 200-400 mg/day in 14 cases, mofetil mycophenolate 1-2 g/day in 3 cases, methotrexate $7.5-15 \mathrm{mg} / \mathrm{week}$ in 6 cases, cyclosporin A $100 \mathrm{mg} /$ day in 1 case and azathioprine $100-150 \mathrm{mg}$ /day in 5 cases). The median (IQ) ESR and CRP values were respectively $13.0(25) \mathrm{mm}^{\text {st }}$ $\mathrm{h}$ and $2.2(3.7) \mathrm{mg} / \mathrm{L}$ and overall median creatinine levels were within the normal range $(0.78 \mathrm{mg} / \mathrm{dL}$; IQ 0.33$)$. The median mRSS score was 5 (IQ 9) with a range between 1 and 30, and values higher than 20 were reported only in 5 cases. Digital ulcers were actively present in $16(23.1 \%)$ patients and were managed with IV prostanoids and topical medications; calcinosis in $11(15.9 \%)$ patients; $11(15.9 \%)$ patients 
were at risk of $\mathrm{PAH}$, detected by means of 2D-ECHO and $30(43.4 \%)$ patients had lung fibrosis, detected by means of chest HRTC. Pulmonary function was globally preserved [median (IQ) DLCO/VA 86\% (20); median (IQ) VC 98\% (20)]. DLCO was below $80 \%$ in 25 patients, but in the majority of the cases fibrosis affected less than $20 \%$ of lung parenchyma and DLCO reduction was in general due to the concomitant PAH. No significant association between lower DLCO values and lung pulmonary fibrosis or PAH has been found $(\mathrm{p}>0.05)$. On the contrary, $\mathrm{VC}$ was below $70 \%$ only in 2 cases and significantly lower in SSc patients with lung fibrosis $(\mathrm{p}=0.001$, 95\%CI 5.15-19.17).

Additionally, we obtained the nailfold capillaroscopic images at the time of recruitment from 42 patients, which were scored according to Cutolo et al. (6). Eight of them (19\%) had a normal pattern, $7(16 \%)$ an early SSc pattern, 16 (38\%) an active
SSc pattern and $11(26 \%)$ a late SSc pattern. Being a qualitative assessment, the capillary pattern was considered as a dicothomous variable (normal versus pathological). Finally, 23 (33.3\%) patients suffered from osteoporosis and 20 of them were taking calcium supplements and cholecalciferol.

The demographic and clinical characteristics of the SSc cohort are reported in Table I.

In the group of SSc patients, the median concentration of serum klotho was $0.23 \mathrm{ng} /$ $\mathrm{mL}$ (IQ 0.61, range 0.04-1.85) with a nonGaussian distribution, while in the control group, mean serum klotho concentration was $0.59 \pm 0.39 \mathrm{ng} / \mathrm{mL}$, with a normal distribution. When descriptive statistical tests were performed on the global cohort, a non-Gaussian distribution of overall klotho values was obtained, after which we chose non parametric tests for the following analyses. Using a two-tailed Mann Whitney

Table I - Demographic and clinic characteristics of the cohort of scleroderma patients.

\begin{tabular}{|l|c|}
\hline SSc patients, n. & 69 \\
\hline Age (years), mean \pm SD & $64.5 \pm 12.5$ \\
\hline Gender (F/M), n. (\%) & $61 / 8(88.4 \% / 11.5 \%)$ \\
\hline Disease duration (years), median, IQ; range & 9,$8 ; 1-57$ \\
\hline Clinical form (limited/diffuse), n.(\%) & $50 / 19(72.4 \% / 27.5 \%)$ \\
\hline ANA+, n. (\%) & $64(92.7 \%)$ \\
\hline Anti-CENB+, n. (\%) & $34(49.2 \%)$ \\
\hline Anti-SCL70+, n. (\%) & $17(24.6 \%)$ \\
\hline ESR (1 h mm), median, IQ; range & 13,$25 ; 2-84$ \\
\hline CRP (mg/L), median, IQ; range & $2.2,3.7 ; 0-24$ \\
\hline Serum creatinine (mg/dL), median, IQ; range & $0.78,0.33 ; 0.49-2.22$ \\
\hline Digital ulcers, n. (\%) & $16(23.1 \%)$ \\
\hline Calcinosis, n. (\%) & $11(15.9 \%)$ \\
\hline PAH, n. (\%) & $11(15.9 \%)$ \\
\hline Lung fibrosis, n. (\%) & $30(43.4 \%)$ \\
\hline DLCO/AV (\%), median, IQ; range & 86,$20 ; 49-118$ \\
\hline FVC (\%), median, IQ; range & 98,$20 ; 58-138$ \\
\hline Medsger's Scale Score, median, IQ; range & $4,2.5 ; 0-12$ \\
\hline mRSS, median, IQ; range & 5,$9 ; 1-30$ \\
\hline Osteoporosis, n. (\%) & $23(33.3 \%)$ \\
\hline
\end{tabular}

SSc, systemic sclerosis; SD, standard deviation; IQ, interquartile interval; F/M, female/male; ANA, antinuclear antibodies; ESR, erythrocyte sedimentation rate; CRP, C-reactive protein; PAH, pulmonary artery hypertension; DLCO/AV, diffusing capacity/transfer factor of the lung for carbon monoxide per unit alveolar volume; FVC, forced vital capacity; mRSS, modified Rodnan's skin score. 
$\mathrm{U}$ test for unpaired samples, a significant reduction in serum klotho concentration in patients compared to controls was detected $(\mathrm{p}<0.001)$, Figure 1.

However, the cohorts of patients and controls significantly differed for gender $(p<0.001)$ and age $(p<0.001)$. Global median concentration of serum klotho was $0.27 \mathrm{ng} / \mathrm{mL}$ (IQ 0.61; range 0.00-1.85) in females and $0.63 \mathrm{ng} / \mathrm{mL}$ (IQ 0.68 , range $0.00-1.70$ ) in males, with a significant difference between males and females $(\mathrm{p}<0.001)$. Among controls, median serum klotho concentrations were lower in females (median values $0.45 \mathrm{ng} / \mathrm{mL}$; IQ 0.46 , range 0.00-1.10) than males (median values $0.71 \mathrm{ng} / \mathrm{mL}$, IQ 0.51, range 0.00-1.70), although this difference was not statistically significant (2-tailed T-test for unpaired samples; $p=0.27$ ); on the contrary, in the patients' group median klotho serum concentrations were higher in females (median values $0.24 \mathrm{ng} / \mathrm{mL}$; IQ 0.66, range 0.04 1.85 ) than males (median values $0.19 \mathrm{ng} /$ $\mathrm{mL}$, IQ 0.15 , range $0.13-0.37$ ) and this difference was statistically significant (twotailed Mann Whitney U test for unpaired samples; $p=0.02$ ). Age minimally affected the concentration of klotho in the overall population $(\mathrm{p}=0.05 ; \mathrm{F} 3.89 ; \mathrm{p}=0.26)$, although not reaching significance when the group of SSc patients and controls were analysed separately $(\mathrm{p}=0.47$; F 0.52 ; $\mathrm{p}=0.008$ for $\mathrm{SSc}$ patients and $\mathrm{p}=0.48$; F $0.49 ; p=0.007$ for controls).

In the group of SSc patients, Spearman correlation showed no significant association between klotho serum levels and disease activity, concerning either clinical, laboratory and instrumental findings.

\section{DISCUSSION}

Klotho is a $130 \mathrm{kDa}$ protein synthetized in many tissues as a soluble, secreted or single-pass trans-membrane form, initially considered as an anti-aging factor. Three genes are responsible for the codification of the $\alpha, \beta$ and $\gamma$ isoforms of klotho. $\alpha$-klotho is mainly produced in the arterial tree, skin, kidney and gut epithelia, neuronal cells and endocrine tissues (7). $\beta$-klotho, sharing a

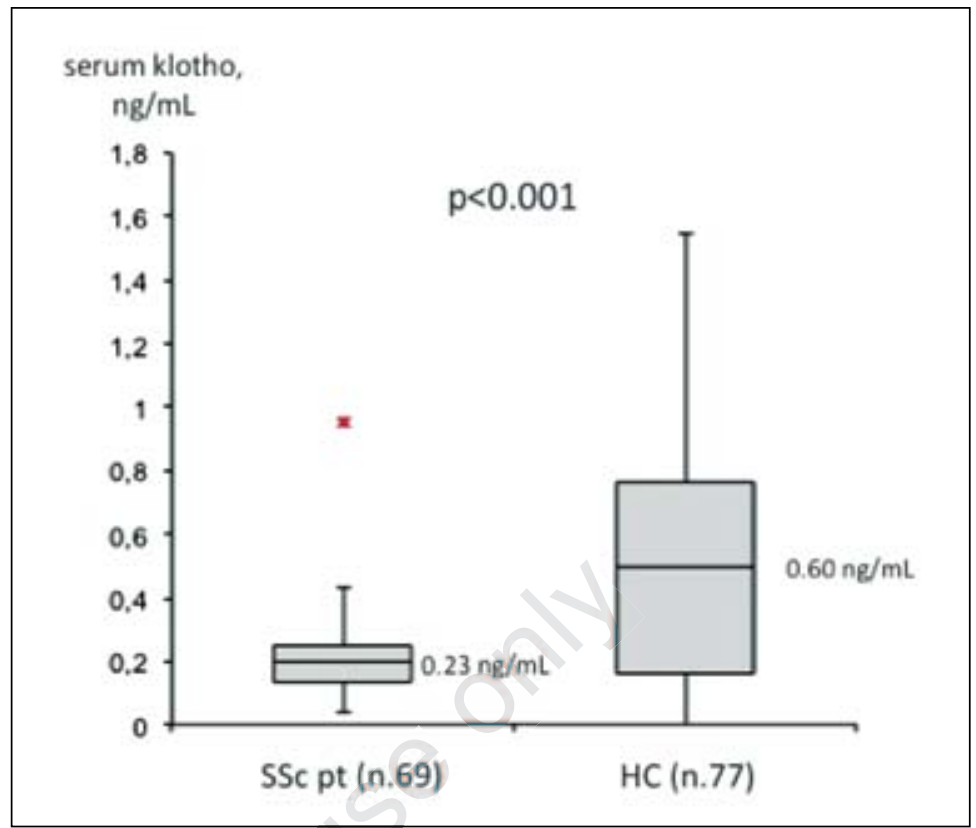

Figure 1 - Median serum concentration of klotho in systemic sclerosis patients and healthy controls. 2-tailed Mann Whitney $U$ test for unpaired sample. SSc pt, systemic sclerosis patients; HC, healthy controls.

$41 \%$ amino acid homology with $\alpha$-klotho, is expressed in adipose tissue, liver and pancreas (8). The entire molecule of klotho contains a single-pass trans-membrane domain and two extracellular domains, known as KL1 and KL2 domains and, following a mechanism of alternative splicing, a secreted isoform of klotho, containing only the KL1 domain, may be generated and released in the extracellular environment. Furthermore, once expressed on the cellular surface, the extracellular KL1 and KL2 domains may be cleaved by secretases and released as soluble klotho (9). Trans-membrane klotho may interact with receptors on neighbouring cells, acting in a paracrine axis, whereas soluble klotho may circulate in the bloodstream and exert its effects on distant organs as an endocrine factor. The KL1 and KL2 domains of both transmembrane and soluble klotho display $\beta$-glucuronidase and sialidase activity by removing glucidic residuals from transient receptor potential vanilloid receptor-related (TRPV5) and TRP canonical-1 (TRPC-1), thus regulating calcium $(\mathrm{Ca})^{2+}$ ions influx into epithelial and endothelial 
cells (10). A deficiency in klotho may reduce the internalization into vascular cells of the complex TRPC-1/vascular endothelium growth factor receptor (VEGFR) that usually follows stimulation with VEGF, with a subsequent increase in the amount of intracellular $\mathrm{Ca}^{2+}$ finally responsible for the $\mathrm{Ca}^{2+}$-dependent activation of calpain and caspase- 3 , which promote endothelial permeability and cell disruption (10). Similarly, klotho may regulate the influx of sodium and potassium in cardiac, renal and nervous cells.

Soluble and trans-membrane klotho may also act as coreceptor, recognizing and strengthening the interaction between fibroblast growth factor (FGF) members and their receptors. $\alpha$-klotho may complex with FGF23 and FGFR1c in the proximal tubules of the kidney and suppress the $1 \alpha$-hydroxylation of $25(\mathrm{OH})^{2}$ vitamin $\mathrm{D}$, thus reducing $\mathrm{Ca}^{2+}$ and phosphate resorption. The excessive concentration of phosphate favours the constriction of vessels and the generation of reactive oxygen species (ROS). A group of researchers demonstrated that the addition in vitro of soluble klotho to human smooth vascular cells or human umbilical vein endothelial cells contrasts phosphate- and FGF23mediated vasoconstriction, by increasing the production of nitric oxide (NO) (11). Furthermore, the authors demonstrated in the same experiment that the addition of soluble klotho without FGF23 to mouse aorta rings induced vasoconstriction and the production of ROS. The increase in endothelial NO following soluble klotho administration could explain the antagonistic effects of klotho to monocrotalineinduced experimental PAH in murine models (12). However, the evaluation of soluble $\alpha$-klotho in the peripheral blood from 64 precapillary PAH patients did not find a significant association with haemodynamic findings (13). It suggests that soluble klotho exerts its hormonal effects modulating the vessel tone according to the presence or not of FGF23 and that $\alpha$ - and $\beta$-klotho may have different roles in controlling myo-contractile cells and in preserving endothelial integrity. Moreover, it has been demonstrated that $\beta$-klotho, but not $\alpha$-klotho, complexes with FGF19 and FGFR4 expressed in the biliary tract, controlling bile acid synthesis (14). $\beta$-klotho may account for the glucidic and lipidic balance, reducing insulin resistance and visceral adiposity, and contributing therefore to minimize vascular damage through different biologic mechanisms. A deficit of klotho has been associated with oxidative stress and mitochondrial damage, which represent specific hallmarks of the endothelial dysfunction in SSc, and to the modulation in the expression of p53 and p21 that accelerates the cell-cycle arrest in G1 phase and cell senescence, generating oxidative stress and autophagy (9). Moreover, soluble klotho binds to several Wnt proteins inhibiting their biological activity, including keratinocyte ageing and connective tissue fibrosis. Klotho-/mice have a short lifespan and an elderly phenotype characterized by a deficit in osteoclasts and osteoblasts, diffuse bone demineralization and vascular tree calcifications. It has been reported that a deficit in human klotho may account for severe tumoral calcinosis (15). Subcutaneous calcinosis is a common finding in SSc and a deficit in serum klotho concentration could be responsible for $\mathrm{Ca}^{2+}$ lumps. Moreover, a deficit in klotho could induce a delay in cutaneous ulcer healing related to a reduced apposition of collagen 1 and 3 in wound tissue (3). However, although SSc patients often suffer from prolonged cutaneous ulcer healing times, the reduced production of collagen observed in klothodeficient mice contrasts with the aberrant deposition of extracellular matrix in SSc. A recent experiment on human primary dermal microvascular endothelial cells and dermal fibroblasts showed that klotho may control the expression of genes involved in tissue repair and angiogenesis, counteracting the aberrant production of extracellular matrix and favouring neovessel sprouting (16). The possible pathway presided by $\alpha$ - and $\beta$-klotho is illustrated in Figure 2.

Our data demonstrate that the median serum concentration of klotho was sig- 
nificantly lower in SSc patients than in healthy controls. These results are in accordance with the hypothesis that a deficit of klotho may account for many manifestations of the disease, including ulcer healing delay, lung fibrosis, calcinosis, renal crisis. Although a standardised range for serum klotho is unavailable, our results are in line with serum klotho levels shown in previous studies on human healthy subjects $(17,18)$. However, due the consecutive enrolment and the design of the study, the cohort of patients significantly differed from controls with respect to gender and age. Klotho concentrations were lower in patients than in controls both in males and females, with the lower concentrations in male patients. Gender may have represented a bias for our analysis; nevertheless, SSc may play an additional role in influencing klotho serum levels, altering the male/female ratio in the group of patients. However, two previous studies measuring serum klotho concentration through an ELISA kit in healthy subjects did not suggest a significant influence of gender on klotho serum concentration $(17,19)$. Furthermore, other studies on non-scleroderma-patient populations reported higher serum klotho concentrations in female than in male patients $(20,21)$. These data are in line with our results, and may suggest that chronic diseases, including SSc, induce an imbalance of the serum klotho concentration with a more pronounced effect on male patients.

On the contrary, age did not affect the overall concentration of serum klotho in both cohorts.

Unfortunately, to our knowledge, no evaluation of soluble klotho in the sera of patients affected by connective tissue diseases has been reported until today. A reduced concentration of $\alpha$-klotho in the cerebrospinal fluid has been associated to neuropsychiatric systemic lupus erythematosus (NSLE) in a multivariate analysis on 84 neuropsychiatric patients (22). Similarly, an impairment in genetic expression and a deficient $\beta$-glucuronidase activity of klotho has been demonstrated in CD4+ T lymphocytes from rheumatoid arthritis (RA)

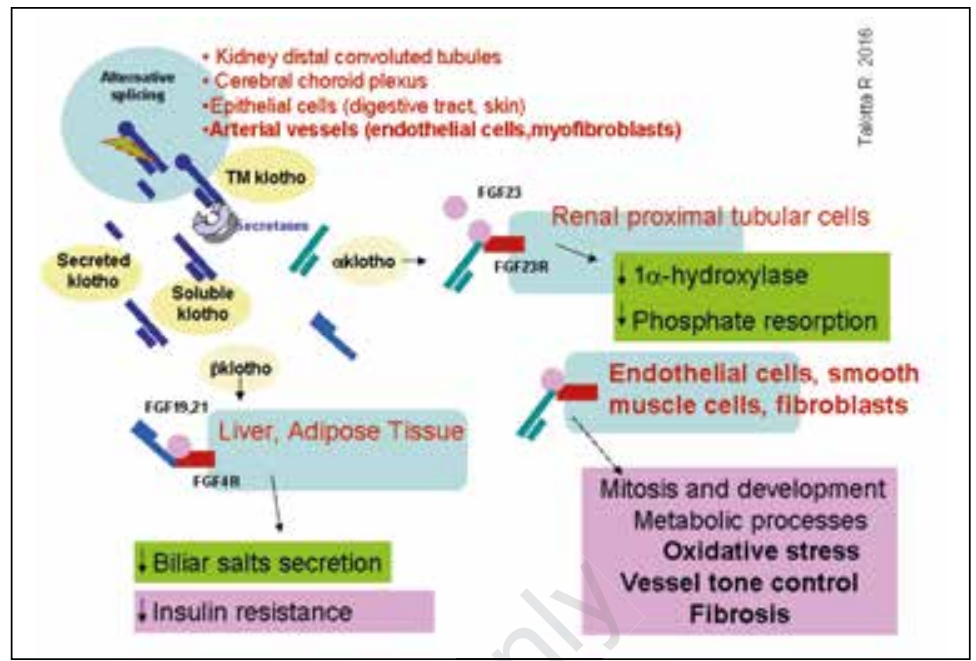

Figure 2 - Biologic pathways presided by $\alpha$ and $\beta$-klotho. Klotho is mainly synthetized in renal distal convolute tubules, cerebral choroid plexus, epithelial cells and vascular tree, as a $130 \mathrm{kD}$ peptide containing a transmembrane domain and two extracellular domains. Following a mechanism of alternative splicing, a short form of secreted klotho, having only the KL1 extracellular domain may be produced. Once expressed on the plasma membrane, transmembrane klotho may be cleaved by extracellular secretases in a soluble form ( $\alpha$ - and $\beta$-klotho) that may act as $\beta$-glucuronidase or coreceptor, complexing with FGF and FGFR. $\beta$-klotho is prevalently involved in electrolytic balance, endothelium integrity, vessel tone control and fibrosis prevention. $\beta$-klotho seems to preside to the control of biliar salt secretion and insulin sensitivity. TM, transmembrane; FGF, fibroblast growth factor; FGFR, fibroblast growth factor receptor.

patients, and associated with premature senescence of this pool of cells (23). In a murine model of autoimmune colitis, it has been demonstrated that cytokines tumour necrosis factor (TNF) and interferon- $\gamma$ reduced the transcription of the klotho gene in renal epithelial cells (24).

SSc seems to display many phenotypic aspects compatible with a deficit of klotho. However, we did not find any significant association between the serum concentration of klotho and the likelihood of undergoing a specific manifestation of the disease. The most important limits of this study consist in the number of subjects involved, in the enrolment of patients coming from mismatched cohorts for gender and age, in the measurement of whole soluble klotho (without any distinction between $\alpha$-klotho and $\beta$-klotho), in the lack of evaluation of secreted, soluble and trans-membrane isoforms, and perhaps in the low sensitiv- 
ity of the ELISA test. Polymorphic variants of the klotho gene were not assessed, since they could explain a quantitative or a qualitative deficit of the molecule. Finally, given the central role of klotho in calcium/ phosphate balance and in bone remodelling, the regular intake of calcium and cholecalciferol in 20 osteoporotic patients and the lack of a control group could have added further bias to our analysis, although no significant association was recorded between serum klotho concentration and the odds of having osteoporosis in the SSc cohort. Moreover, a recent work by Chalhoub et al. failed to assign sufficient relevance to soluble klotho as a biomarker of bone resorption and fracture risk (25).

\section{CONCLUSIONS}

Our data show a reduced concentration of serum klotho in patients affected by SSc compared to healthy controls with no significant correlation with clinical, laboratory or instrumental features of the disease. Many experiments in vitro and in vivo have demonstrated an important role of klotho in the control of vessel tone, tissue reparation, ectopic calcifications, electrolytic balance and wound healing, and some of these aspects are shared by SSc. Due to the limits of our study, the results obtained failed to show any significant association with the phenotypic characteristics of the disease and further investigations are indeed needed in order to better understand the real involvement of klotho on the pathogenesis of SSc.

Acknowledgements: we acknowledge the Medical and Nursing Staff of the Rheumatology Unit of the Luigi Sacco University-Hospital for their collaboration in this work.

\section{REEERENCES}

1. Kuro-o M. Overview of the FGF23-Klotho axis. Pediatr Nephrol. 2010; 25: 583-90.

2. Kuro-o M. Klotho as a regulator of fibroblast growth factor signaling and phosphate/calcium metabolism. Curr Opin Nephrol Hypertens. 2006; 15: 437-41.
3. Yamashita K, Yotsuyanagi T, Yamauchi M, Young DM. Klotho mice: a novel wound model of aged skin. Plast Reconstr Surg Glob Open. 2014; 2: e101.

4. van den Hoogen F, Khanna D, Fransen J, et al. 2013 classification criteria for systemic sclerosis: an American college of rheumatology/ European league against rheumatism collaborative initiative. Ann Rheum Dis. 2013; 72: 1747-55

5. Geirsson AJ, Wollheim FA, Akesson A. Disease severity of 100 patients with systemic sclerosis over a period of 14 years: using a modified Medsger scale. Ann Rheum Dis. 2001; 60: 1117-22.

6. Cutolo M, Sulli A, Pizzorni C, Accardo S. Nailfold videocapillaroscopy assessment of microvascular damage in systemic sclerosis. J Rheumatol. 2000; 27: 155-60.

7. Lim K, Groen A, Molostvov G, et al. $\alpha$-Klotho expression in human tissues. J Clin Endocrinol Metab. 2015; 100: E1308-18.

8. Ito S, Kinoshita S, Shiraishi N, et al. Molecular cloning and expression analyses of mouse betaklotho, which encodes a novel Klotho family protein. Mech Dev. 2000; 98 : 115-9.

9. Bian A, Neyra JA, Zhan M, Hu MC. Klotho, stem cells, and aging. Clin Interv Aging. 2015; 10: $1233-43$.

10. Kusaba T, Okigaki M, Matui A, et al. Klotho is associated with VEGF receptor-2 and the transient receptor potential canonical- $1 \mathrm{Ca} 2+$ channel to maintain endothelial integrity. Proc Natl Acad Sci USA. 2010; 107: 19308-13.

11. Six I, Okazaki H, Gross P, et al. Direct, acute effects of Klotho and FGF23 on vascular smooth muscle and endothelium. PLoS One. 2014; 9: e93423.

12. Varshney R, Ali Q, Wu C, Sun Z. Monocrotaline-induced pulmonary hypertension involves downregulation of antiaging protein klotho and eNOS activity. Hypertension. 2016; 68: 1255-63.

13. Kaiser R, Seiler S, Held M, et al. Prognostic impact of renal function in precapillary pulmonary hypertension. J Intern Med. 2014; 275: 116-26.

14. Kurosu H, Kuro-o M. The klotho gene family as a regulator of endocrine fibroblast growth factors. Mol Cell Endocrinol. 2009; 299: 72-8.

15. Ichikawa S, Imel EA, Kreiter ML, et al. A homozygous missense mutation in human KLOTHO causes severe tumoral calcinosis. J Clin Invest. 2007; 117: 2684-91.

16. Markiewicz M, Panneerselvam K, Marks N. Role of Klotho in migration and proliferation of human dermal microvascular endothelial cells. Microvasc Res. 2016; 107: 76-82.

17. Pedersen L, Pedersen SM, Brasen CL, Rasmussen LM. Soluble serum Klotho levels in healthy subjects. Comparison of two differ- 
ent immunoassays. Clin Biochem. 2013; 46: 1079-83.

18. Mostafidi E, Moeen A, Nasri H, et al. Serum Klotho levels in trained athletes. Nephrourol Mon. 2016; 8: e30245.

19. Yamazaki Y, Imura A, Urakawa I, et al. Establishment of sandwich ELISA for soluble alpha-Klotho measurement: age-dependent change of soluble alpha-Klotho levels in healthy subjects. Biochem Biophys Res Commun. 2010; 398: 513-8.

20. Semba RD, Cappola AR, Sun K, et al. Plasma klotho and cardiovascular disease in adults. J Am Geriatr Soc. 2011; 59: 1596-601.

21. Sze L, Neidert MC, Bernays RL, et al. Gender dependence of serum soluble Klotho in acromegaly. Clin Endocrinol (Oxf). 2014; 80: 869-73.
22. Ushigusa T, Ichinose K, Sato S, et al. Soluble $\alpha$-klotho is a potential biomarker associated with neuropsychiatric systemic lupus erythematosus. Clin Immunol. 2016; 165: 29-34.

23. Witkowski JM, Soroczyńska-Cybula M, Bryl $\mathrm{E}$, et al. Klotho-a common link in physiological and rheumatoid arthritis-related aging of human CD4+ lymphocytes. J Immunol. 2007; 178: 771-7.

24. Thurston RD, Larmonier CB, Majewski $\mathrm{PM}$, et al. Tumor necrosis factor and interferon-gamma down-regulate Klotho in mice with colitis. Gastroenterology. 2010; 138: 1384-94.

25. Chalhoub D, Marques E, Meirelles O, et al. Association of serum klotho with loss of bone mineral density and fracture risk in older adults. J Am Geriatr Soc. 2016; 64: e304-8. 